\title{
Comparing Blood Pressure of Twins and Their Singleton Siblings: Being a Twin Does Not Affect Adult Blood Pressure
}

\author{
Eco J.C. de Geus', Danielle Posthuma', Richard G. IJzerman² and Dorret I. Boomsma' \\ ' Vrije Universiteit Amsterdam, The Netherlands \\ ${ }^{2}$ Academic Hospital Vrije Universiteit Amsterdam, The Netherlands
}

\begin{abstract}
The hypothesis was tested that monozygotic (MZ) and dizygotic (DZ) twins, with their lower average birth weight, have higher adult blood pressure than their singleton brothers or sisters. From the Netherlands Twin Registry, 261 twin families were recruited from a young adult and an older adult cohort with mean ages of 26.2 and 50.4 respectively. These families yielded $204 \mathrm{MZ}$ twins with 71 singleton siblings and $271 \mathrm{DZ}$ twins with 103 of their singleton siblings. Anti-hypertensive medication use of these 649 participants was assessed twice with a two-year interval. Resting blood pressure was measured thrice during a standardized laboratory protocol. In spite of a significant difference in birth weight (1036 gram), no differences were found in anti-hypertensive medication use at both time points between twins and singletons nor between their resting laboratory diastolic or systolic blood pressure. These results applied to each gender and to both age cohorts. Limiting the analyses to matched twin-sibling pairs of the same families and taking current weight and height into account did not change the results; no evidence was found for a twin-singleton difference. It was concluded that estimates of genetic and environmental contributions to blood pressure deriving from twin studies do not appear to be biased and may be generalized to singletons. Our results suggest that the lower birth weight in twins does not reflect the intrauterine disadvantage described by the Barker hypothesis.
\end{abstract}

A systematic review of the literature on the relation between birth weight and adult blood pressure showed that newborns with low birth weight are at increased risk for hypertension (Huxley et al., 2000). It has proven difficult to establish specific intrauterine factors explaining this association or, more generally, to prove that the association derives from a causal effect of intrauterine factors at all (Paneth \& Susser, 1995; Walker et al., 1998). For instance, social class of the mother, including its effects on nutritional behavior and smoking habits, may independently influence birth weight and hypertensive risk (Ben-Shlomo \& Smith, 1991; Williams \& Poulton, 1999). Genetic susceptibility of the mother to hypertension or insulin resistance may cause her to have deviant physiology during pregnancy which in turn influences birth weight in the offspring. High blood pressure in the offspring could then reflect the inheritance of maternal susceptibility to hypertension and/or insulin resistance rather than low birth weight (Churchill et al., 1997;
Hattersley \& Tooke, 1999; Walker et al., 1998). Also, pleiotropic genes may be inherited from either parent that influence fetal growth as well as adult blood pressure.

An optimal design to exclude confounding of socioeconomic status and to reduce that of genetic factors is to compare the blood pressure of adult twins to that of their singleton brothers and sisters. Twins have, on average, much lower birth weights than singletons (Bleker et al., 1988). This lower birth weight remains after correction for gestational age and is based mainly on retarded growth in the third trimester (Ananth et al., 1998; Glinianaia et al., 2000). Both $\mathrm{MZ}$ and $\mathrm{DZ}$ twins share the family environment and $50 \%$ of their genes with their singleton brothers and sisters. Because the parental genes influencing blood pressure will segregate randomly to twins and singletons, genetic effects will not influence the mean difference in blood pressure in large groups of twin-singleton pairs. Likewise, higher average blood pressure in twins compared to their singleton siblings cannot be due to shared family environment like the parental socioeconomic class. It could, when found, be attributed to the special intrauterine condition of a twin, which is reflected so clearly in their lower birth weight. This finding would be in line with the "Barker hypothesis" (Barker, 1998) that attributes the association between low birth weight and high adult blood pressure to a causal effect of adverse intrauterine factors.

Apart from providing a well-controlled test of the association of birth weight to adult blood pressure, the comparison of twin-singleton differences in adult blood pressure has important ramifications outside the realm of the Barker hypothesis. In genetic epidemiology, heritability of blood pressure and changes in heritability across different environments (gene-environment interaction) are usually assessed by comparing MZ and DZ twins (Martin et al., 1997). A causal effect of low birth weight or other twin-specific intrauterine aspects on later blood pressure would imply

Address for correspondence: EJC De Geus, Vrije Universiteit, Department of Biological Psychology, Van der Boechorststraat 1, 1081 BT, Amsterdam, The Netherlands. Email: eco@psy.vu.nl 
that heritability estimates from twin studies can not be generalised to the singleton population.

The present study will test the hypothesis that twins have higher blood pressure than their singleton brothers or sisters. In 261 twin families of which 156 included at least one singleton sibling, resting blood pressure was measured in a standardized laboratory setting. Because high blood pressure is now often detected early and successfully treated, differences in antihypertensive medication use between singleton and twin populations may mask effects of twinning on blood pressure. Twin-singleton differences in antihypertensive use, therefore, were also tested.

\section{Method \\ Participants}

Data were collected from two cohorts of twin families that participated in a large ongoing project on the genetics of adult brain function (Posthuma et al., 2000; Posthuma et al., in press). All subjects underwent screening to exclude cases of pathology known to affect brain structure, including vascular brain trauma. Heart disease or hypertension per se, however, were no exclusion criteria.

Six hundred and forty-nine family members from a total of 261 extended twin families participated in this study. The sample was divided in two cohorts (young adults mean age 26.2 years and older adults mean age 50.4 years) based on the age of the twins. There was a slight overlap in age of the non-twin siblings between the two cohorts. In both cohorts zygosity was tested by DNA polymorphisms.

In the young cohort 123 twin pairs, 19 single twins and 101 additional siblings participated. In the older cohort 98 twin pairs, 14 single twins and 73 additional siblings participated.

The study was approved by the Vrije Universiteit Ethics Committee, and all subjects gave written informed consent prior to their participation.

\section{Measurements}

Twins and singleton siblings came to the laboratory to undergo extensive testing for cognitive functioning including reaction time tests and electroencephalographic recording. Most sessions took place between 9 am and 2 pm, with late afternoon sessions for an occasional subject. There was no difference in the mean time of day for blood pressure measurements for twins $(0: 26 \mathrm{pm})$ and singletons $(0: 41$ $\mathrm{pm})$. Throughout the measurement session participants were sitting in a recliner chair in a sound and dimly lit cabin. After an acclimatization period and instructions on the EEG procedures blood pressure was taken three times with a 2-minute interval during quiet rest. The average of the three blood pressure values is used in the analyses. Blood pressure measurements were taken from an arm-cuff on the left arm by an automated blood pressure device (Dinamap 845XT, Critikon Inc) using an oscillometric technique to estimate SBP and DBP. Throughout the resting period the researcher remained outside of the cabin.

About two years before testing all participants had filled out a questionnaire on personality, lifestyle and health, which included a number of questions on medication use. This questionnaire was again administered at the time of the laboratory measurements. Two different types of medication use were distinguished: antihypertensive medication (ACE inhibitors, beta-blockers) and cardioactive medication in a broad sense (antihypertensives, lipid lowering drugs, drugs influencing electrical cardiac signal conduction, insulin, repeated use of aspirin or other antithrombotic treatment).

Hundred twenty six of the twins in the young cohort had previously participated in a study on the genetics of cardiovascular risk. A significant association of birth weight and blood pressure in this original twin sample, after adjustment for gender and current weight, has previously been reported on (Ijzerman et al., 2000). From these 126 twins, birth weight data were obtained from the mother, when the family visited our laboratory (twins were aged 16 at that time). A maternal questionnaire on birth weight was sent to the mothers a few weeks ahead of their visit to our department, allowing them to obtain birth data from birth certificates. Birth weight of additional 139 young twins and singleton siblings and 160 older twins and singleton siblings were obtained by questionnaire to the participants themselves. We attempted, were possible, to improve reliability of these questionnaire data by telephone interview. In 224 subjects, however, no reliable self-report of BW could be obtained so far, most often because the parents had died or could not be tracked.

During the laboratory session body weight and height were assessed with the subjects wearing only light clothing. Height was measured to the nearest centimeter, with subjects standing on a hard surface against a wall, using a square and tape measure fixed to the wall. Body weight was measured to within $100 \mathrm{~g}$ using a regularly calibrated scale. BMI was calculated as the weight in kilograms divided by the square of height in meters.

\section{Statistical Analysis}

MANOVA (GLM SPSSwin 10) was used to compare the systolic and diastolic blood pressure of the twins and singletons. Although the differences in birth weight between MZ and $\mathrm{DZ}$ are less striking than those between twins and singletons (van Baal \& Boomsma, 1998) we tested for possible blood pressure differences between MZ and DZ twins as well. Thus, a first between subject factor Zygosity had three levels: $\mathrm{MZ}, \mathrm{DZ}$ or singleton. Gender was entered as a second, and age cohort as a third between subject factor. The Zygosity (3) x Gender (2) x Age cohort (2) MANOVA was performed twice: with and without BMI as covariate. Finally, all analyses were redone using only participants without any antihypertensive or cardioactive medication use.

The difference in percentage antihypertensive or cardioactive medication use between $\mathrm{MZ} / \mathrm{DZ} /$ singletons was tested with separate Chi-square tests in subgroups of young males, older males, young females and older females.

\section{Results}

Tables 1 and 2 show the subject characteristics and resting blood pressure for the male and female twins and singletons in both age cohorts. MANOVA showed the expected significant effect of zygosity on birth weight $(F(2,424)=$ $84.2, p<0.0001)$; no further significant main or interaction effects involving gender or cohort were found. Tukey 
post-hoc testing showed a significant contrast between singleton and dizygotic twin birth weight (947 gram) as well as between dizygotic and monozygotic birth weight (212 gram).

To test whether the inverse relation between blood pressure and birth weight also held in this population a stepwise regression was performed of gender (dummycoded), age, weight, length and birth weight on blood pressure. This was done separately in twins and singletons. A substantial proportion of the variance of SBP and DBP was predicted in twins (SBP, $R^{2}=.54$; DBP, $R^{2}=.45$ ) as well as singletons (SBP, $R^{2}=.59$; DBP, $R^{2}=.49$ ). In twins birth weight significantly contributed to adult SBP level as shown in the upper panel of table 3. An inverse relation between systolic blood pressure and birth weight was also apparent in singletons, but did not reach significance, possibly due to the smaller sample size (lower panel table 3). Birth weight did not contribute to the prediction of DBP in either group.

\section{Twin Singleton Comparisons}

Almost no medication was used by the younger subjects, but $\chi^{2}$ tests in the older cohort showed no difference in either gender in antihypertensive use or the use of other medication for cardiovascular conditions. $\mathrm{MZ}$ and $\mathrm{DZ}$ twins did not differ from each other and neither differed from singletons.

MANOVA on the diastolic and systolic variates showed a significant effect of age cohort $(F(2,638)=81.0, p<$ $0.0001)$ and gender $(F(2,638)=9.90, p<0.0001)$. Tukey post-hoc analyses revealed that, as expected, SBP and DBP were higher in males than in females, and higher in older than in younger participants. A further univariate age cohort by gender interaction for DBP was found $(F(1,639)$ $=9.23), p<0.002$ ) due to a larger age related increase in DBP in males than in females. No other effects on SBP or DBP were found. Specifically, the comparison of twins and singletons did not yield any significant main or interaction effects. When the analyses on blood pressure were repeated excluding all medication users, again no twin singleton differences were found.

\section{Table 1}

Subject Characteristics, Medication Use, and Mean (Standard Deviation) of Resting Blood Pressure — Young Cohort

\begin{tabular}{|c|c|c|c|c|c|c|}
\hline \multirow[b]{3}{*}{ Age } & \multicolumn{3}{|c|}{ Male } & \multicolumn{3}{|c|}{ Female } \\
\hline & $\begin{array}{l}\text { MZ twin } \\
(N=49)\end{array}$ & $\begin{array}{l}\mathrm{DZ} \text { twin } \\
(N=66)\end{array}$ & $\begin{array}{l}\text { Singleton sibling } \\
\qquad(N=49)\end{array}$ & $\begin{array}{l}\mathrm{MZ} \text { twin } \\
(N=58)\end{array}$ & $\begin{array}{l}\mathrm{DZ} \text { twin } \\
(N=92)\end{array}$ & $\begin{array}{l}\text { Singleton sibling } \\
\qquad(N=52)\end{array}$ \\
\hline & (3.0) & $25.7 \quad(2.4)$ & (6.8) & (3.1) & (2.7) & $27.3 \quad(5.9)$ \\
\hline BMI & (2.6) & $23.7 \quad(3.6)$ & $24.3 \quad(3.7)$ & (3.2) & (2.8) & $23.2 \quad(3.6)$ \\
\hline Weight & $73.1 \quad(10.3)$ & $77.7 \quad(11.5)$ & $80.0 \quad(13.7)$ & $63.5 \quad(10.3)$ & $65.7 \quad(10.0)$ & $67.8 \quad(11.6)$ \\
\hline Birth weight & $\begin{array}{c}2541 \quad(588) \\
(n=38)\end{array}$ & $\begin{array}{c}2581 \quad(494) \\
(n=55)\end{array}$ & $\begin{array}{c}3558 \quad(449) \\
(n=18)\end{array}$ & $\begin{array}{c}2344 \quad(613) \\
(n=44)\end{array}$ & $\begin{array}{c}2553 \quad(635) \\
(n=77)\end{array}$ & $\begin{array}{c}3508 \quad(564) \\
(n=33)\end{array}$ \\
\hline Antihypertensives ( $M$ ) & 0 & 0 & 0 & 0 & 1 & 1 \\
\hline CVD medication $(M)$ & 0 & 0 & 0 & 0 & 1 & 1 \\
\hline Resting SBP (mmHg) & $119.9 \quad(12.2)$ & $127.1 \quad(9.7)$ & $124.3 \quad(10.9)$ & 118.9 & 118.7 & $119.2 \quad(10.3)$ \\
\hline Resting DBP (mmHg) & $71.4 \quad(11.4)$ & $74.1 \quad(8.7)$ & $73.7 \quad(9.9)$ & $72.2 \quad(7.0)$ & $72.8 \quad(7.3)$ & $73.1 \quad(7.9)$ \\
\hline
\end{tabular}

\section{Table 2}

Subject Characteristics, Medication Use, and Mean (Standard Deviation) of Resting Blood Pressure — Older Cohort

\begin{tabular}{|c|c|c|c|c|c|c|c|}
\hline \multirow[b]{3}{*}{ Age } & \multicolumn{4}{|c|}{ Male } & \multicolumn{3}{|c|}{ Female } \\
\hline & \multicolumn{2}{|c|}{$\begin{array}{c}\mathrm{MZ} \\
(N=46)\end{array}$} & $\begin{array}{c}\mathrm{DZ} \\
(N=45)\end{array}$ & $\begin{array}{l}\text { Singleton } \\
(N=34)\end{array}$ & $\begin{array}{c}\mathrm{MZ} \\
(N=51\end{array}$ & $\begin{array}{c}\mathrm{DZ} \\
(N=68)\end{array}$ & $\begin{array}{l}\text { Singleton } \\
(N=39)\end{array}$ \\
\hline & 49.1 & $(7.0)$ & $51.4 \quad(6.8)$ & $51.1 \quad(8.7)$ & (7.4) & $50.2 \quad(6.8)$ & $48.3 \quad(9.0)$ \\
\hline BMI & 25.8 & $(2.9)$ & $26.7 \quad(3.7)$ & $26.5 \quad(2.6)$ & $(4.2)$ & 25.6 & 26.7 \\
\hline Weight & 84.1 & $(9.8)$ & $87.3 \quad(16.5)$ & $86.4 \quad(9.6)$ & $68.4 \quad 12.1)$ & $69.9 \quad(13.4)$ & $73.1 \quad(16.1)$ \\
\hline Birth weight & $\begin{array}{r}2389 \\
\quad(n=\end{array}$ & $\begin{array}{l}(751) \\
26)\end{array}$ & $\begin{array}{c}2685 \quad(925) \\
(n=22)\end{array}$ & $\begin{array}{c}3526 \quad(522) \\
(n=51)\end{array}$ & $\begin{array}{c}2264 \quad(736) \\
(n=36)\end{array}$ & $\begin{array}{c}2594 \quad(633) \\
(n=44)\end{array}$ & $\begin{array}{c}3566 \quad(674) \\
(n=19)\end{array}$ \\
\hline Antihypertensives $(M)$ & & & 6 & 3 & 3 & 8 & 4 \\
\hline CVD medication ( $N$ ) & & & 7 & 4 & 7 & 11 & 6 \\
\hline Resting SBP (mmHg) & 137.5 & $(15.1)$ & $133.3 \quad(12.7)$ & $133.6 \quad(12.8)$ & $131.1 \quad(16.1)$ & $132.4 \quad(16.9)$ & $128.9 \quad(19.1)$ \\
\hline Resting DBP (mmHg) & 86.1 & $(10.7)$ & $83.2 \quad(11.3)$ & $(7.7)$ & $79.4 \quad(11.4)$ & $80.2 \quad(10.7)$ & $79.3 \quad(10.1)$ \\
\hline
\end{tabular}


Table 3

Stepwise Regression of Systolic Blood Pressure on Gender (Dummycoded), Age, Weight, Length and Birth Weight in Twins (Upper Panel) and Singletons(Lower Panel). (The Table Lists the Unstandardized $\beta$ 's with Confidence Intervals and Significance.)

\begin{tabular}{|c|c|c|c|c|c|}
\hline Predictor & $\beta$ & $\mathrm{T}$ & $p$ & $\begin{array}{l}\text { Confidence } \\
\text { intervals }\end{array}$ & $\beta$ \\
\hline \multicolumn{6}{|l|}{ Twins $(N=337)$} \\
\hline Gender & -4.677 & -2.903 & .004 & -7.847 & -1.508 \\
\hline Age & .406 & 8.142 & .000 & .308 & .504 \\
\hline Length (m) & -14.557 & -1.464 & .144 & -34.122 & 5.008 \\
\hline Weight (kg) & .184 & 3.510 & .001 & .081 & .287 \\
\hline Birth weight (kg) & -1.829 & -2.103 & .036 & -3.539 & -.118 \\
\hline \multicolumn{6}{|l|}{ Singletons $(N=88)$} \\
\hline Gender & -1.668 & -.485 & .629 & -8.520 & 5.185 \\
\hline Age & .301 & 3.026 & .003 & .103 & .499 \\
\hline Length $(\mathrm{m})$ & 4.071 & .210 & .834 & -34.546 & 42.688 \\
\hline Weight (kg) & .355 & 3.395 & .001 & .147 & .564 \\
\hline Birth weight (kg) & -1.253 & -.598 & .552 & -5.428 & 2.922 \\
\hline
\end{tabular}

In contrast, MANOVA on BMI index showed, in addition to the expected main effects of gender $(F(1,637)=6.44$, $p<0.011)$ and age cohort $(F(1,637)=102.5, p<0.0001)$, a main effect of twin-singleton status $(F(2,637)=6.51, p<$ 0.002). Tukey post-hoc comparison showed significantly lower $\mathrm{BMI}$ in the $\mathrm{MZ}$ twins in comparison with singletons, but no differences between $\mathrm{DZ}$ twins and $\mathrm{MZ}$ twins or $\mathrm{DZ}$ twins and singletons. Separate testing of weight and height showed the BMI effect to be entirely due to differences in weight, with height virtually identical in $\mathrm{MZ}$ twins, DZ twins and singletons. When the analyses of variance on blood pressure were repeated 1) with BMI and age as covariates and 2) additionally excluding all medication users, again no $\mathrm{DZ}$ versus $\mathrm{MZ}$ or twin versus singleton differences were found.

Finally, we computed all possible twin-singleton differences in resting blood pressure within each of the families that had at least one singleton sibling. In same-sex twin families, the mean blood pressure of the twin was subtracted from the mean value of all same-sex singletons. In oppositesex twins the mean of the male twin was subtracted from the mean value of all male singletons, and the mean of the female twin from the mean value of the female singletons. Figure 1 shows these twin singleton differences for SBP and DBP as a function of the age of the twin. No significant correlation of these differences to age - which would have indicated increasing twin-singleton differences with aging - was found in either type of twin or gender. Again no evidence for a blood pressure disadvantage in twins, i.e. a deviation from zero, was apparent at any age.

\section{$\overline{\text { Discussion }}$}

In spite of the well-known differences in birth weight, these data provide no evidence for an increased risk of hypertension in twins compared to singletons. No differences were

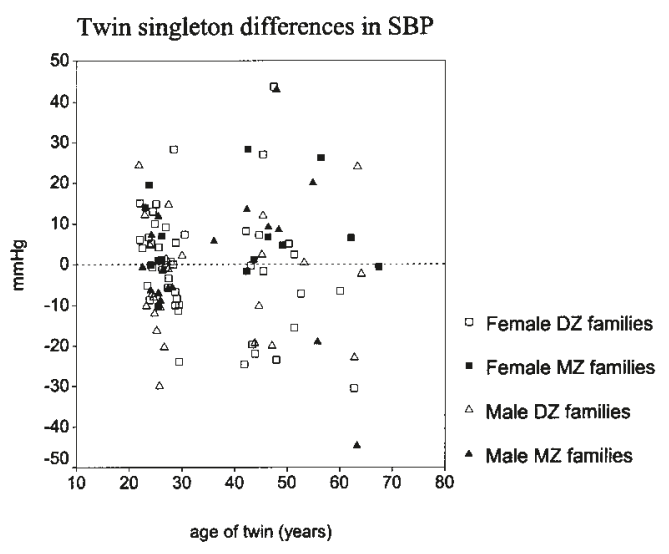

Twin singleton differences in DBP

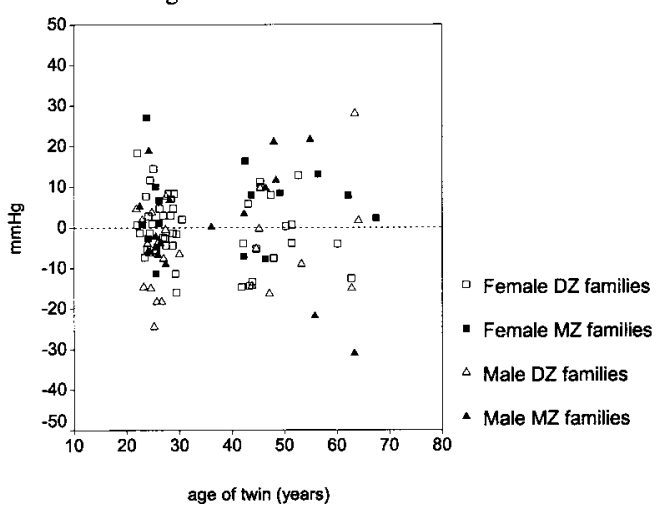

Figure $\mathbf{1 a}$ and $\mathbf{1 b}$.

Within family twin-singleton differences for systolic blood pressure (SBP) and diastolic blood pressure (DBP) as a function of the age of the twin. Values are given separately for the four gender by zygosity groups.

found in antihypertensive medication use at both time points or in their resting laboratory diastolic or systolic blood pressure. These results applied to both genders. Also, no evidence was found for a selective increase in hypertensive risk in the older cohort of twins, or any indication of a widening gap between twins and their singleton brothers or sisters with age. This would have been predicted from studies showing an increase of the association between birth weight and blood pressure with increasing age (Huxley et al., 2000). In accordance with findings in the large Finnish adolescent twin cohort, singletons had higher BMI than twins (Pietalinen et al., 1999) which was entirely due to higher singleton body weight. Correcting for either weight or BMI, however, left the findings unchanged: no twin-singleton differences in blood pressure were found. The absence of twin-singleton differences in blood pressure or antihypertensive use is in full agreement with the lack of evidence for increased CVD mortality among twins in comparison to singletons (Christensen et al., 1995; Vagero \& Leon, 1994).

Our findings have clear ramifications for studies using twins to establish the genetic architecture of blood pressure regulation. A causal effect of low birth weight or other 
twin-specific intrauterine aspects on later blood pressure could have compromised heritability estimates from twin studies. A shared intrauterine disadvantage may not only increase the mean blood pressure of twins but also its variance relative to the variance of singletons, and increase the covariance between twins in comparison to the sibling covariance. It would have been difficult to predict the impact of such "twinning" effects on estimates of genetic and environmental contribution to interindividual differences in blood pressure. In the worst case scenario, a twin singleton difference would have nullified the advantage of an increase in statistical power when singleton siblings are added to twins in a so-called "extended twin design" (Posthuma \& Boomsma, 2000). Fortunately, our results suggest that estimates of genetic and environmental contributions to blood pressure deriving from twin studies do not appear to be biased and may be generalized to singletons.

The inverse relation between birth weight and adult blood pressure repeatedly found in singletons (Martyn et al., 1995; Zureik et al., 1996) was recently found to also hold in a large population of $\mathrm{DZ}$ and $\mathrm{MZ}$ twins, part of which also participated in the current study (IJzerman et al., 2000). Our results now replicate this finding in a larger sample of twins. At the same time no difference is found between mean singleton and twin blood pressure, in spite of an average difference in birth weight of 1036 grams. The absence of a twin-singleton blood pressure difference in this withinfamily study seems, at first glance, to contradict the Barker hypothesis that low birth weight causes high blood pressure. However, in the Barker hypothesis, reduced birth weight is used as an index of "retarded intrauterine growth" or "placental insufficiency". The culprit is not birth weight per se, but adverse aspects of intrauterine life (e.g. nutritional deficiencies) that cause high blood pressure through impaired renal development (Brenner \& Chertow, 1994; Lackland et al., 2000), low fetal vessel compliance (Martyn et al., 1995) or inadequate HPA-axis function (Philips et al., 2000). It is uncertain that the low birth weight in twins compared to their singleton siblings reflects the same impairment in fetal growth that is reflected in the birth weight differences in singletons. The low birth weight in twin compared to singleton pregnancies largely reflects reduced growth in the third trimester (Bleker et al., 1988) probably due to reduced deposition of soft tissue (Kuno et al., 1999; Xu et al., 1995). This may well represent a healthy physiological adaptation to the increased energy demands of a twin pregnancy (Kuno et al., 1999) that has no consequences in later life. Our results support this with regard to blood pressure. The absence of a twin-singleton difference clearly shows that the lower birth weight of twins does not reflect the intrauterine disadvantage described by the Barker hypothesis.

In short, the present findings neither support nor refute the Barker hypothesis. It should be pointed out, nonetheless, that our data are compatible with alternative explanations of the birth weight to blood pressure relationship. Birth weight not just reflects intra-uterine factors but also the genetic make-up of fetus and mother as well as a host of postnatal familial factors like SES related dietary or other health practices of the family. These genetic and environmental factors could well affect adult blood pressure in addition to their effects on birth weight. A number of studies suggest that social class of the mother only slightly attenuates the relation between adult cardiovascular risk and birth weight (Leon et al., 1998). Genetic effects on birth weight and blood pressure do exist, however, as became apparent in two studies in twins. IJzerman et al. (2000) compared intra-pair differences in $\mathrm{MZ}$ and $\mathrm{DZ}$ twins and found significantly lower regression of $\mathrm{MZ}$ than $\mathrm{DZ}$ intrapair differences in birth weight on blood pressure during rest and mental challenge. These results further demonstrated that elimination of genetic factors strongly attenuates the relation between birth weight and blood pressure. A large twin study by Poulter et al. (1999) also supports a genetic explanation, although their own conclusions were to the opposite. In a study of $\mathrm{MZ}$ and $\mathrm{DZ}$ twins, they found no significant association between intra-pair differences in blood pressure and intra-pair differences in birth weight in $\mathrm{MZ}$ twins. Because $\mathrm{MZ}$ twins have identical genes, no such association is expected if genetic effects are important. However, DZ twins did have a significant association of intra-pair differences in blood pressure and birth weight as shown in a re-analyses of their data (IJzerman et al., 2000).

Genes common to blood pressure and birth weight can work in two ways: First, maternal "blood pressure" genes may influence quality of pregnancy in the mother but also, when passed on, blood pressure in the offspring. Genes influencing blood pressure through their effects on obesity, a very heritable trait, are good examples. Obesity in the mother affects placental to birth weight ratio during pregnancy (Clarson et al., 1989; Perry et al., 1995) that has been found to be a strong predictor of adult blood pressure (Barker et al., 1990). Part of this prediction may derive from passing on the obesity genes, and the higher risk for high blood pressure they convey, to the offspring. Secondly, pleiotropic genes from either father or mother may influence offspring birth weight as well as adult blood pressure. Genetic pleiotropy assumes that genes affecting adult hypertensive risk will also affect the birth weight of offspring. For instance, insulin secreted by the fetal pancreas in response to maternal glucose concentrations is a key growth factor. Genetically determined insulin resistance may result in impaired insulin-mediated growth in the fetus as well as insulin resistance in adult life (Hattersley \& Tooke, 1999). Finally, intra-uterine "Barker-like" effects may interact with genetic factors as suggested by the finding that low birth weight most strongly predicts blood pressure in subjects who become obese (Leon et al., 1996), where obesity is known to be determined largely by genetic endowment (Comuzzie \& Allison, 1998).

The current evidence from twin studies does not favor intrauterine factors, as suggested by the Barker hypothesis, as the main explanation for a birth weight to adult blood pressure association. Multivariate analyses of twin families with multiple siblings targeting both blood pressure and birth weight must answer the critical questions: to what extent is the observed correlation between blood pressure and birth weight due to familial factors and to what extent are these environmental and genetic in nature. 


\section{Acknowledgements}

We gratefully acknowledge the financial support of the Vrije Universiteit USF (grant number 96/22) and the Human Frontiers of Science Program Organization (grant number $\operatorname{rg} 0154 / 1998-B)$.

\section{References}

Ananth, C. V., Vintzileos, A. M., Shen-Schwarz, S., Smulian, J. C., \& Lai, Y. L. (1998). Standards of birth weight in twin gestations stratified by placental chorionicity. Obstetrics and Gynecology, 91, 917-924.

Barker, D. J. (1998). In utero programming of chronic disease. Clinical Science, 95, 115-128.

Barker, D. J., Bull, A. R., Osmond, C., \& Simmonds, S. J. (1990). Fetal and placental size and risk of hypertension in adult life. British Medical Journal, 301, 259-262.

Ben-Shlomo, Y., \& Smith, G. D. (1991). Deprivation in infancy or in adult life: Which is more important for mortality risk? Lancet, 337, 530-534.

Bleker, O. P., Oosting, J., \& Hemrika, D. J. (1988). On the cause of the retardation of fetal growth in multiple gestations. Acta Geneticae Medicae et Gemellologiae, 37, 41-46.

Brenner, B. M., \& Chertow, G. M. (1994). Congenital oligonephropathy and the etiology of adult hypertension and progressive renal injury. American Journal of Kidney Disease, 23, $171-175$.

Christensen K., Vaupel, J. W., Holm, N. V., \& Yashin, A. I. (1995). Mortality among twins after age 6: Fetal origins hypothesis versus twin method. British Medical Journal, 18, 432-436.

Churchill, D., Perry, I. J., \& Beevers, D. G. (1997). Ambulatory blood pressure in pregnancy and fetal growth. Lancet, 349, 7-10.

Clarson, C., Tevaarwerk, G. J., Harding, P. G., Chance, G. W., \& Haust, M. D. (1989). Placental weight in diabetic pregnancies. Placenta, 10, 275-281.

Comuzzie, A. G., \& Allison, D. B. (1998). The search for human obesity genes. Science, 280, 1374-1347.

Glinianaia, S. V., Skjaerven, R., \& Magnus, P. (2000). Birthweight percentiles by gestational age in multiple births. A populationbased study of Norwegian twins and triplets. Acta Obstetricia et Gynecologica Scandinavica, 79(6), 450-458.

Hattersley, A. T., \& Tooke, J. E. (1999). The fetal insulin hypothesis: An alternative explanation of the association of low birth weight with diabetes and vascular disease. Lancet, 353, 1789-1792.

Huxley, R. R., Shiell, A. W., \& Law, C. M. (2000). The role of size at birth and postnatal catch-up growth in determining systolic blood pressure: A systematic review of the literature. Journal of Hypertension, 18, 815-831.

IJzerman, R. G., Stehouwer, C. D. A., \& Boomsma, D. I. (2000). Evidence for genetic factors explaining the birth weight-blood pressure relation. Analysis in twins. Hypertension, 36, 1008-1012.

Kuno, A., Akiyama, M., Yanagihara, T., \& Hata, T. (1999). Comparison of fetal growth in singleton, twin, and triplet pregnancies. Human Reproduction, 14(5), 1352-1360.
Lackland, D. T., Bendall, H. E., Osmond, C., Egan, B. M., \& Barker, D. J. (2000). Low birth weights contribute to high rates of early-onset chronic renal failure in the Southeastern United States. Archives of Internal Medicine, 160, 1472-1476.

Leon, D. A., Koupilova, I., Lithell, H. O., Berglund, L., Mohsen, R., Vagero, D., Lithell, U. B., \& McKeigue, P. M. (1996). Failure to realise growth potential in utero and adult obesity in relation to blood pressure in 50 year old Swedish men. British Medical Journal, 312, 401-406.

Leon, D. A., Lithell, H. O., Vagero, D., Koupilova, I., Mohsen, R., Berglund, L., Lithell, U. B., \& McKeigue, P. M. (1998). Reduced fetal growth rate and increased risk of death from ischaemic heart disease: Cohort study of 15000 Swedish men and women born 1915-29. British Medical Journal, $317,241-245$.

Martin, N., Boomsma, D., \& Machin, G. (1997). A twin-pronged attack on complex traits. Nature Genetics, 17, 387-392.

Martyn, C. N., Barker, D. J., Jespersen, S., Greenwald, S., Osmond, C., \& Berry, C. (1995). Growth in utero, adult blood pressure, and arterial compliance. British Heart Journal, 73(2), 116-121.

Paneth, N., \& Susser, M. (1995). Early origins of coronary heart disease (the Barker hypothesis). British Medical Journal, 310, 411-412.

Perry, I. J., Beevers, D. G., Whincup, P. H., \& Bareford, D. (1995). Predictors of ratio of placental weight to fetal weight in multiethnic community. British Medical Journal, 310, 436-439.

Phillips, D. I., Walker, B. R., Reynolds, R. M., Flanagan, D. E., Wood, P. J., Osmond, C., Barker, D. J., \& Whorwood, C. B. (2000). Low birth weight predicts elevated plasma cortisol concentrations in adults from 3 populations. Hypertension, 35 , 1301-1306.

Pietilainen, K. H., Kaprio, J., Rissanen, A., Winter, T., Rimpela, A., Viken, R. J., \& Rose R. J. (1999). Distribution and heritability of BMI in Finnish adolescents aged 16y and 17y: A study of 4884 twins and 2509 singletons. International Journal of Obesity and Related Metabolic Disorders, 23(2), $107-115$.

Posthuma, D., \& Boomsma, D. I. (2000). A note on the statistical power in extended twin designs. Behavior Genetics, 30(2), 147-158.

Posthuma, D., de Geus, E. J., Bleichrodt, N., \& Boomsma, D. I. (2000). Twin-singleton differences in intelligence? Twin Research, 3, 83-87.

Posthuma, D., de Geus, E. J. C., Smith, G., Boomsma, D. I. (2001). Genetic correlation between inspection time and IQ. Behavior Genetics, (in press). 
Poulter, N. R., Chang, C. L., MacGregor, A. J., Snieder, H., \& Spector, T. D. (1999). Association between birth weight and adult blood pressure in twins: Historical cohort study. British Medical Journal, 19, 1330-1333.

Vagero, D., \& Leon, D. (1994). Ischaemic heart disease and low birth weight: A test of the fetal-origins hypothesis from the Swedish Twin Registry. Lancet, 29, 260-263.

van Baal, C. G., \& Boomsma, D. I. (1998). Etiology of individual differences in birth weight of twins as a function of maternal smoking during pregnancy. Twin Research, 1, 123-30.

Walker, B. R., McConnachie, A., Noon, J. P., Webb, D. J., \& Watt, G. C. (1998). Contribution of parental blood pressures to association between low birth weight and adult high blood pressure: Cross sectional study. British Medical Journal, $316,834-837$.

Williams, S., \& Poulton, R. (1999). Twins and maternal smoking: Ordeals for the fetal origins hypothesis? A cohort study. British Medical Journal, 318, 1-5.

Xu, B., Deter, R. L., Milner, L. L., \& Hill, R. M. (1995). Evaluation of twin growth status at birth using individualized growth assessment: Comparison with conventional methods. Journal of Clinical Ultrasound, 23(5), 277-286.

Zureik, M., Bonithon-Kopp, C., Lecomte, E., Siest, G., \& Ducimetiere, P. (1996). Weights at birth and in early infancy, systolic pressure, and left ventricular structure in subjects aged 8 to 24 years. Hypertension, 27, 339-345. 